\title{
Swarms of diversity at the gene cox 1 in Antarctic krill
}

\author{
WP Goodall-Copestake ${ }^{1}$, S Pérez-Espona ${ }^{2}$, MS Clark ${ }^{1}$, EJ Murphy ${ }^{1}$, PJ Seear ${ }^{1}$ and GA Tarling ${ }^{1}$ \\ ${ }^{1}$ British Antarctic Survey, Natural Environment Research Council, Cambridge, UK and ${ }^{2}$ School of Biological Sciences, University \\ of Bristol, Bristol, UK
}

\begin{abstract}
The Antarctic krill, Euphausia superba, is an abundant and key species found in the Southern Ocean that forms dense, discrete swarms. Despite over three decades of research on Antarctic krill, the genetics of individual swarms is yet to be specifically investigated. In this study, we address the genetic diversity, population structure and demographic history of nine Antarctic krill swarms by sequencing 1173 bases of the gene cytochrome $c$ oxidase subunit I (cox1, COI) from 504 individuals. Both haplotype diversity $(h=0.9974-1.0000)$ and nucleotide diversity $(\pi=0.010275-0.011537)$ of Antarctic krill swarm samples was consistently high compared with populations of other species reported in the literature. Analysis of
\end{abstract}

molecular variance did not show any significant genetic structure, thus implying that the sampled swarms do not appear to reflect discrete genetic units. Fu's Fs and Bayesian Skyride analyses provided strong evidence for a large increase in the population size of Antarctic krill, or selection favouring a particular mitochondrial lineage, within the last few 100000 years (Pleistocene). The swarm-level results presented in this study not only further our understanding of Antarctic krill biology but, because of the economical importance of this species, also provide data to consider for future krill stock management. Heredity (2010) 104, 513-518; doi:10.1038/hdy.2009.188; published online 20 January 2010

Keywords: COI; Euphausia superba; haplotype diversity; population genetics; Southern Ocean; stock management

\section{Introduction}

The Antarctic krill, Euphausia superba is one of the most renowned biomass-rich organisms found in the oceans, with estimates for the standing stock of approximately $1-4 \times 10^{8}$ tonnes comprising $1-10 \times 10^{14}$ post-larval individuals (Atkinson et al., 2009). The abundance of Antarctic krill makes it a key species in the Antarctic food web and, increasingly, an important resource for fisheries. Antarctic krill often occur within dense swarms characterized by uniform length-frequency distributions (Watkins, 1986) and synchronized cycles of moulting and spawning (Buchholz et al., 1996). Marr (1962) considered such non-random distribution of krill to start from the larval stage and continue through development, with each swarm being a separate entity that is 'discrete and complete in itself'. This suggests a little mixing between swarms, although such inter-swarm discrimination would seem difficult to maintain, particularly when swarms disperse to feed at night (Godlewska, 1996).

The population genetics of Antarctic krill has been studied since the 1970s, initially using allozyme variation (c.f. Fevolden and Schneppenheim, 1989) and most recently using mitochondrial DNA sequences (Zane et al., 1998). Despite advances in our knowledge attributable to these studies, the genetics of Antarctic krill at the level of the swarm is yet to be investigated.

Correspondence: Dr WP Goodall-Copestake, British Antarctic Survey (NERC), High Cross, Madingley Road, Cambridge CB3 OET, UK.

E-mails:wogco@bas.ac.uk

Received 27 August 2009; revised 25 November 2009; accepted 14

December 2009; published online 20 January 2010
One reason for this is that sampling individual krill swarms is not trivial, as swarm limits need to be defined and separate swarms individually sampled. In contrast, krill swarms used in previous studies were often obtained on an opportunistic basis from multi-year collections and, consequently, it was unclear whether they represented discrete swarms, a number of swarms in close proximity or even the same swarm sampled more than once. Given that swarms are often considered as the fundamental unit of krill biology, it is crucial to study the population genetics of krill at this level.

In this study, we address the genetic diversity, population structure and demographic history of nine discrete Antarctic krill swarm samples collected from the Scotia Sea, a region that contains a large fraction of total Antarctic krill biomass and occupies a central position within its distributional range (Atkinson et al., 2009). We used the mitochondrial gene cytochrome $c$ oxidase subunit I ( $\operatorname{cox} 1, \mathrm{COI})$ as a molecular marker because it is routinely used for intra-specific studies on invertebrates, and sequence accessions held on GenBank show that cox1 is sufficiently variable to assess the intra-species diversity of Antarctic krill. Furthermore, cox1 has been shown to be suitable as a barcode for krill species verification (Bucklin et al., 2007). Adult Antarctic krill are distinctive and readily identifiable, in particular because of their large size of up to $64 \mathrm{~mm}$ measuring from the eye to the telson (Atkinson et al., 2008). However, species identification from small juvenile krill is more challenging, and therefore DNA barcoding is an important tool for verifying the identity of such material.

In this study, we also assessed the quality of our cox 1 data set by evaluating the reproducibility of our DNA 
sequencing procedure and by using comparative analyses to check for the presence of contaminating nuclearmitochondrial DNA (numts), as both factors have been shown to bias evolutionary genetic analyses severely (Clark and Whittam, 1992; Buhay, 2009). In addition, we applied a new 'Bayesian Skyride' method to infer the demographic history of swarms. This method allows for weaker prior assumptions and seems to show less error over a range of possible demographic histories than do other 'classical' and 'Bayesian Skyline' methods (Minin et al., 2008). The use of Bayesian Skyride analysis will shed light on the demographic history of krill, a subject of great interest, following reports on the decline of Antarctic krill stocks over recent decades (1976-2003) that may be linked to climate change (Atkinson et al., 2008).

\section{Materials and methods}

\section{Antarctic krill sampling}

Samples for genetic analysis were obtained during a single oceanographic cruise carried out by the British Antarctic Survey (January-February 2003) in the Scotia Sea, Southern Ocean. A rectangular midwater trawl (RMT8; 8- $\mathrm{m}^{2}$ mouth area, 2-mm mesh) was used to sample krill from discrete swarms located with an echosounder (Simrad EK60, Simrad, Horten, Norway). Specimens were immediately frozen at $-80^{\circ} \mathrm{C}$ upon capture. Further details on sampling and preservation methods are described in the study by Tarling et al. (2006). We considered a total of nine swarm-specific net samples, collected from successive locations along the cruise track (Figure 1). Importantly, the time window over which the samples were collected was only 15 days; hence, we can assume that the same krill swarm was not inadvertently sampled more than once, given the distance between sampling events and the speed of ocean currents (c.f. Thorpe et al., 2004). A total of 56 krill per swarm sample were obtained for further genetic analysis.

\section{DNA extraction}

Abdominal muscle was used for DNA extraction, as this is rich in mitochondria and likely to contain fewer parasites than other mitochondria-rich tissues such as

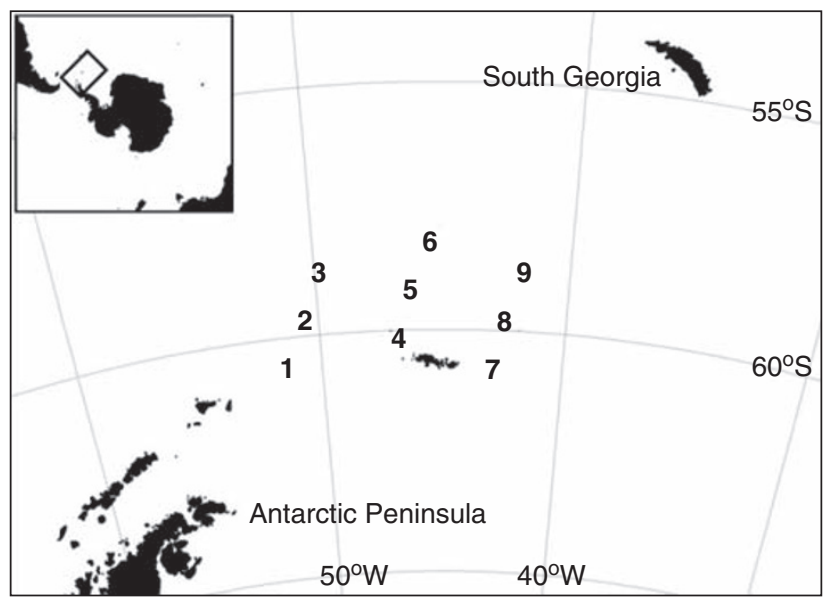

Figure 1 Sampling locations of Antarctic krill swarms, labelled numerically in order of the sampling. The total time between samples 1 and 9 was 15 days (12-27 January 2003). gills. Total genomic DNA was extracted directly from frozen tissue using a DNeasy Tissue Kit (Qiagen, Hilden, Germany), including the optional step to degrade RNA. Successful extraction of DNA depended on procedures being directly applied to the frozen tissue, because we experienced rapid DNA degradation within seconds of thawing. Furthermore, it was necessary to include RNAase as Antarctic krill contain large quantities of RNA that seems to saturate DNA-binding columns at the expense of DNA.

\section{cox1 DNA sequencing}

PCR amplification was tailored to amplify mitochondrial cox1 rather than numts by using species-specific cox1 PCR primers, a low amount of template DNA and stringent PCR conditions. The species-specific primers were designed using the near complete Antarctic krill mitochondrial genome (GenBank accession no. AB084378) to amplify a 1303 base region of cox 1 . The $5^{\prime}$ to $3^{\prime}$ sequences of the newly designed primers are: AB084378_1294: CTCAACTAATCACAAAGACATTGG and AB084378_2596: ACCCGATAGAGGATAATACATT TC. PCR reactions were $25 \mu \mathrm{l}$ and contained $1 \times$ PCR buffer, $1.3 \mathrm{mM} \mathrm{MgCl}_{2}, 0.2 \mathrm{mM}$ dNTPs, $0.2 \mu \mathrm{M}$ each primer, 0.7 Units BioTaq (Bioline, London, UK) and c. $5 \mathrm{ng}$ template DNA. The thermocycling profile consisted of $1 \mathrm{~min}$ at $95^{\circ} \mathrm{C}$, then 30 cycles of $20 \mathrm{~s}$ at $95^{\circ} \mathrm{C}, 1 \mathrm{~min}$ at $61^{\circ} \mathrm{C}$ and $3 \mathrm{~min}$ at $72^{\circ} \mathrm{C}$.

Amplified DNA fragments were purified using an EXO-SAP method (GE Healthcare, Little Chalfont, UK). Cycle sequencing was conducted using a Big Dye Terminator cycle sequencing kit v.3.1 (Applied Biosystems, Foster City, CA, USA), using the same primers as used for initial amplification, together with an additional novel internal primer AB084378_1609: 5'-GAAGAGGTT TAGTAGAAAGTGG-3'. Sequencing products were run on a MegaBACE 1000 automated DNA sequencer (GE Healthcare). The software CodonCode Aligner v.2.01 (CodonCode, Dedham, MA, USA) was used to edit electropherograms and generate consensus sequence reads 1173 bases long for each krill sample. Ambiguities in these consensus reads were resolved by re-amplifying and re-sequencing cox 1 . All of the unique sequences generated were deposited in GenBank (accession numbers GQ305462-GQ305901).

\section{Investigating sequencing error}

To investigate possible sequencing error, we estimated the reproducibility of our sequencing procedure. One DNA sample was randomly selected from each krill swarm sample (total $n=9$ ) and the entire PCR amplification, cycle sequencing and electropherogram editing procedure was repeated nine times (that is, a total of 81 PCR amplifications and 243 sequence reads to generate 81 consensus sequences).

\section{DNA sequence data sets}

Two DNA alignments were generated (A and B), both without alignment ambiguity, gaps or missing data. The main data set, alignment A, consisted of all 504 fulllength (1173 base) sequences obtained from the nine krill swarm samples. An additional data set, alignment B, was generated from the sequences in alignment $\mathrm{A}$ together with cox1 GenBank accessions for Antarctic krill 
(AB084378), Euphausia crystallorophias (AF177183) and Euphausia vallentini (AF177188), as well as for two Antarctic krill cox1 cDNA sequences (unpublished data, PJS). The Antarctic krill cox1 GenBank accession and cDNA sequences were included for comparative tests for numts, and the other accessions were included for tests of species membership: the Antarctic E. crystallorophias was used because it appears to be the sister species of $E$. superba, and the sub-Antarctic E. vallentini was included as an out-group (c.f. Patarnello et al., 1996). After alignment, trimming and removal of identical sequences, alignment B consisted of 292 sequences that were 579 bases long.

\section{DNA substitution modelling}

The majority of variable sites in DNA alignments A and $B$ occurred at the third codon positions with only a few variable sites at the first position (see the 'Results' section below). To model this variability, we followed the rationale and approach of Kaliszewska et al. (2005), by generating a data partition from each alignment containing all third position sites augmented by the variable sites at the first position (data partitions $\mathrm{A}^{*}$ and $\mathrm{B}^{*}$ ). DNA sequence variability in these data partitions was then evaluated for nucleotide homogeneity using tetrahedral plots and matched-pairs tests for symmetry (Ababneh et al., 2006) implemented in the software SeqVis v.1.3 (Ho et al., 2006). Significant compositional heterogeneity was inferred if the dots in the tetrahedral plots were widely dispersed and if $x \%$ of the matched-pairs tests of symmetry produced $P$-values $\geqslant x$ (that is, $x>5 \%$ at $P=0.05, x>1 \%$ at $P=0.01)$. Finally, the software $\mathrm{jMo}-$ deltest v.0.1.1 (Guindon and Gascuel, 2003; Posada, 2008) was used to select an appropriate substitution model for each partition using maximum likelihood optimized trees and Akaike and Bayesian information criteria selection procedures.

\section{Comparative assessment for numts and species membership}

DNA alignments $\mathrm{A}$ and $\mathrm{B}$ were translated using a mitochondrial invertebrate code and screened for stop codons and (numerous) amino-acid changes, as these are indicative of numts. As a further test for numts and to test for species membership, we reconstructed phylogenies from the data in partition $\mathrm{B}^{*}$. We used the maximum likelihood non-parametric bootstrapping method implemented in the software GARLI v.0.96 (Zwickl, 2006) with the best-fitting model obtained from jModeltest applied and 1000 bootstrap replicates. Genuine Antarctic krill mitochondrial $\operatorname{cox} 1$ sequences would be expected to form a well-supported monophyletic group with the Antarctic krill GenBank accession and cDNAs.

\section{Population diversity and structure analyses}

The software ARLEQUIN v.3.1 (Excoffier et al., 2005) was used to calculate descriptive statistics from alignment A on the basis of the raw number of differences between sequences, these included haplotype diversity ( $h$; Nei, $1987)$ and nucleotide diversity ( $\pi$; Nei and Li, 1979). To increase the comparative value of our results, descriptive statistics were re-calculated using the first 600 bases of DNA alignment $\mathrm{A}$ as this approximately corresponds with the most frequently sequenced region of cox1.
Analysis of molecular variance (AMOVA; Excoffier et al., 1992) was used to test for genetic structure among krill swarms. For the AMOVA analysis, $\Phi_{\mathrm{ST}}$ was estimated using ARLEQUIN and data partition $A^{*}$. One group of nine populations was defined, and pairwise distances were imported from maximum likelihood treebased estimates calculated using GARLI according to the selected model of DNA substitution. Analyses based solely on haplotype frequencies were not conducted because of the low number of shared haplotypes in the data set (see the 'Results' section below). A total of 10000 nonparametric permutations of the data, computed using ARLEQUIN, were used to generate significance values.

\section{Historical demography analyses}

The historical demography of Antarctic krill was investigated by calculating Fu's Fs (Fu, 1997), which has been shown to be the most powerful commonly used test for detecting population size change when sample sizes are large (Ramos-Onsins and Rozas, 2002). Fs was calculated using ARLEQUIN from alignment A with 10000 coalescent simulation samples to test for statistical significance. We also estimated population size trajectories using a new Bayesian Skyride method implemented in the software BEAST v.1.5beta3 (Drummond and Rambaut, 2007). The analyses were run using data partition $\mathrm{A}^{*}$, a Gaussian Markov random field smoothing prior (Minin et al., 2008), the selected model of DNA substitution and a strict molecular clock. No direct Antarctic krill intra-species calibration rates are available to calibrate this clock. Therefore, we used a crude pairwise rate of $2 \%$ per million years on the basis of the approximate mid-point of crustacean divergence values in a recent comparison made by Lessios (2008); this rate was scaled before applying it to data partition $\mathrm{A}^{*}$. It is important to note that differences in population history, ecology and DNA substitution modelling between the Antarctic krill individuals studied herein and the samples used to generate the calibration rate will introduce error in population size trajectories. Three Markov chains were run, each starting from a different tree obtained from preliminary analyses that used a constant size model. Proposal operators for chain moves were optimized to generate acceptance rates from 0.1 to 0.4 ; each chain was run for $3 \times 10^{8}$ steps and sampled every 10000 steps. These conditions were selected to generate 'effective sample sizes' $>200$ from chains that appeared to have reached stationarity (see BEAST manual). Analyses were repeated without sequence data to obtain estimates from the prior distribution. Samples from the first $3 \times 10^{7}$ steps of each chain were removed as burnin (which appeared to be sufficient, see BEAST manual) and subsequent samples were compared using the software Tracer v.1.4.1 (Rambaut and Drummond, 2007) and combined to generate the final parameter estimates. A krill generation time (that is, from egg to reproducing adult) of 2 years (Bargmann, 1945) was used to scale estimates for the female effective population size $\left(N_{\mathrm{ef}}\right)$.

\section{Results}

\section{Sequence error}

The reproducibility of our sequencing procedure, assessed by selecting nine DNA samples and regenerating 
the final consensus sequence of each sample nine times, showed one ambiguity corresponding to overlapping $\mathrm{C}$ and $\mathrm{T}$ peaks in a single-pass sequence read. This reflects an overall observable reproducibility error of 1 in 95013 bases and implies a high sequencing accuracy. On the basis of this level of reproducibility, we would expect approximately six ambiguities in the original data set. In fact, 8 ambiguities occurred in 504 consensus sequences originally generated; 1 of these corresponded to overlapping peaks from all 4 nucleotides and the remainder corresponded to overlapping $\mathrm{C}$ and $\mathrm{T}$, or $\mathrm{A}$ and $\mathrm{G}$ peaks. All of these ambiguities were resolved by re-amplifying and re-sequencing cox 1 , and therefore these do not seem to represent mitochondrial heteroplasmy or DNA sample contamination.

\section{Substitution model selection}

Codon positions 1, 2 and 3 contained 18, 0 and 236 variable sites, respectively, in DNA alignment A and 16, 0 and 157 variable sites, respectively, in alignment B. Tetrahedral plots and matched-pairs tests of symmetry did not reveal any significant nucleotide compositional heterogeneity within data partitions $\mathrm{A}^{*}$ and $\mathrm{B}^{*}$, suggesting that they could be analysed using commonly applied time-reversible DNA substitution models. Results obtained from the Akaike and Bayesian information criteria selection procedures consistently showed that a GTR model should provide the best approximation of $\operatorname{cox} 1$ nucleotide substitution.

\section{Comparative assessment for numts and species membership}

Two amino-acid changes were predicted in DNA alignment $\mathrm{A}$, one change in alignment $\mathrm{B}$ and no stop codons were inferred in either of the alignments. Results obtained from the maximum likelihood phylogenetic analysis of data partition $\mathrm{B}^{*}$ showed that all Antarctic krill mitochondrial cox 1 sequences formed a monophyletic group with a bootstrap support of $100 \%$ (data not shown). Taken together, these results provide strong evidence that old or divergent numts were not present in our data sets. The phylogenetic analysis also confirmed that small juvenile krill included in our swarm-specific net samples were Antarctic krill.

\section{Population diversity and structure}

All nine krill swarm samples had similarly large numbers of haplotypes and private haplotypes, high haplotype diversities and high nucleotide diversities
(Table 1); details of the distribution of haplotypes among swarms are provided in the electronic Appendix. After re-calculating the diversity estimates using a smaller 600 base partition of DNA alignment A, values for the number of haplotypes $(n=40-49)$, haplotype diversity $(h=0.9662-0.9942)$ and nucleotide diversity $(\pi=$ 0.009299-0.010907) were only slightly smaller. These recalculated values show that the high diversity estimates in Table 1 are not simply a reflection of the length cox 1 DNA sequenced in this study (1173 bases) compared with the c. 600 bases that are typically sequenced from the $5^{\prime}$ region of cox 1 in other studies.

Results from the AMOVA analysis showed that all genetic variation was within rather than among Antarctic krill swarms. Global and pairwise $\Phi_{\mathrm{ST}}$ values between krill swarm samples were low and not significant (global $\Phi_{\mathrm{ST}}=-0.00136, P=0.56297$; pairwise $\Phi_{\mathrm{ST}}$ ranging from -0.01085 to $0.02145, P>0.05643$ ), therefore indicating a lack of genetic structure between Antarctic krill swarms.

\section{Historical demography}

The value of Fu's Fs was large, negative and significant $(\mathrm{Fs}=-23.62778, P<0.00850)$. Large negative values of $\mathrm{Fs}$ indicate a population expansion of Antarctic krill at the organismal level or an increase in the frequency of a particular mitochondrial lineage within the krill population due to selection or both of these factors. Results from the Bayesian Skyride analysis are presented as a demographic trajectory for $N_{\text {ef }}$ (Figure 2); as is the case with Fs, this could also represent the impact of selection. The trajectory shows strong evidence for an exponential increase in abundance and some indication that this may have been followed by a subtle decrease in abundance, although the evidence for this decrease is weak. Assuming that the demographic trajectory in Figure 2 is genuine and taking molecular clock error into account, we suggest that the most recent common ancestor of the sampled krill cox1 lineages existed in the order of hundreds of thousands of years ago, during the Pleistocene, and that the most recent doubling in krill abundance occurred within the last 100000 years. Bayesian prior estimates showed no change in $N_{\text {ef, }}$ therefore indicating that the posterior estimates reflect a genuine signal in the data and not an artefact of prior assumptions.

\section{Discussion}

The cox1 haplotype diversity of all nine Antarctic krill swarm samples was high (ratio of haplotypes to

Table 1 Descriptive statistics for the cox1 diversity of nine Antarctic krill swarm-specific net samples

\begin{tabular}{lccccc}
\hline Krill swarm & No. individuals & No. haplotypes & No. private haplotypes & Haplotype diversity \pm s.d. & Nucleotide diversity \pm s. $d$. \\
\hline 1 & 56 & 55 & 45 & $0.9994 \pm 0.0035$ & $0.010687 \pm 0.005429$ \\
2 & 56 & 56 & 51 & $1.0000 \pm 0.0034$ & $0.011537 \pm 0.005838$ \\
3 & 56 & 55 & 48 & $0.9994 \pm 0.0035$ & $0.010275 \pm 0.005231$ \\
4 & 56 & 54 & 45 & $0.9987 \pm 0.0037$ & $0.010937 \pm 0.005549$ \\
5 & 56 & 54 & 46 & $0.9994 \pm 0.0035$ & $0.011194 \pm 0.005673$ \\
6 & 56 & 53 & 47 & $0.9974 \pm 0.0037$ & $0.011085 \pm 0.005621$ \\
7 & 56 & 56 & 47 & $1.0000 \pm 0.0034$ & $0.011017 \pm 0.005588$ \\
8 & 56 & 54 & NA & $0.9987 \pm 0.0037$ & $0.010699 \pm 0.005435$ \\
9 & 56 & 440 & & $0.9981 \pm 0.0005$ & $0.011233 \pm 0.005692$ \\
Combined & 504 & & & $0.010952 \pm 0.005467$ \\
\hline
\end{tabular}

Abbreviations: NA, not applicable; s.d., standard deviation.. 


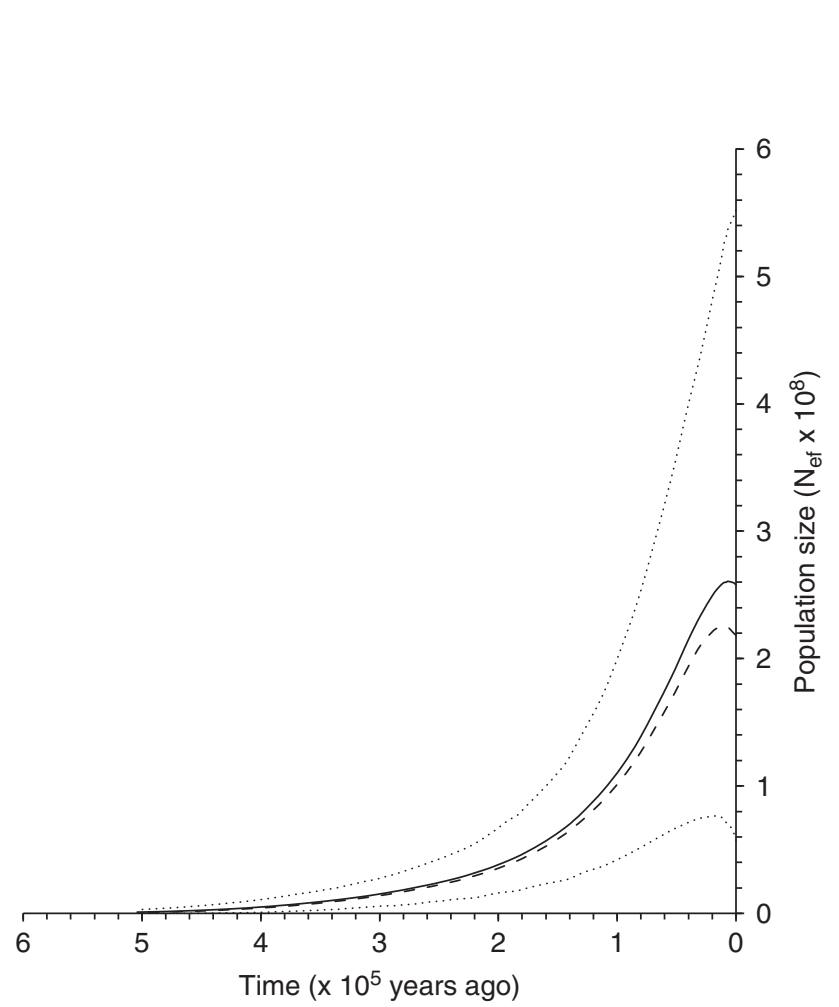

Figure 2 Bayesian Skyride female effective population size $\left(N_{\mathrm{ef}}\right)$ trajectory for Antarctic krill obtained using a pairwise molecular clock rate of $2 \%$ per million years. The mean estimate is shown as a solid line, median estimate as a dashed line and 95\% credibility limits as dotted lines. Mean and median estimates for the most recent common ancestor under this rate were $6.3 \times 10^{5}$ years ago.

individuals $0.95-1.00, h=0.9974-1.0000$, Table 1 ). This result is particularly notable, given that 56 individuals were sampled per swarm and the fact that haplotype diversity is very sensitive to sample size (Nei, 1987). Small sample sizes can easily produce high cox1 haplotype diversities, but high cox1 haplotype diversities are rare in studies with large population sample sizes. The few examples we are aware of that have a sample size of $\geqslant 15$ and a haplotype diversity $>0.99$ come from the marine amphipod Caprella mutica (Ashton et al., 2008), a fig-pollinating wasp Ceratosolen sp. 1 (Lin et al., 2008), the brittle star Ophiothrix fragilis (Muths et al., 2009) and the intertidal gastropods Nerita plicata and Nerita albicilla (Crandall et al., 2008). Of these species, only $N$. plicata has consistently high haplotype diversity over multiple population samples, such as in our case with Antarctic krill. The nucleotide diversity of cox 1 was also consistently high within all krill swarms $(\pi=0.010275-$ 0.011537 , Table 1 ). To our knowledge, only one previous study has found consistently high nucleotide diversity across populations (Cyamus ovalis; Kaliszewska et al., 2005), whereas several studies have reported much higher nucleotide diversity for individual populations, usually from samples of cryptic species or the products of incipient speciation followed by secondary contact (for example, brittle stars, Muths et al., 2009). The high Antarctic krill diversity values reported in this study are unlikely to be an artefact of sequencing error or the presence of numts, as our sequence reproducibility assessment and tests for numts effectively ruled out these possibilities. Furthermore, our re-calculated descriptive statistics showed that the high diversity values did not simply reflect the fact that we analysed a longer region of cox1 compared with other studies (1173 versus 600 bases, respectively). Rather, the high diversity reported in this study seems to be a genuine reflection of the exceptional abundance of Antarctic krill and the potential of the Southern Ocean for gene pool mixing (c.f. Thorpe et al., 2004).

Results obtained from the test for genetic structure suggested that our Antarctic krill swarms samples are genetically mixed and not structured. Thus, the formation of such swarms probably reflects behavioural and/ or environmental sorting rather than shared ancestry. Contrary to the supposition of Marr (1962), it is unlikely that a swarm remains as a discrete unit from the larval to the adult stage, but rather, mixing between swarms is likely to be relatively commonplace. Our results agree with those from previous allozyme-based studies of Antarctic krill in which no genetic structure was found (c.f. Fevolden and Schneppenheim, 1989). In contrast, the only other study using mitochondrial DNA sequences on Antarctic krill (154 bases from the gene ND1) inferred low, yet significant genetic structure (Zane et al., 1998). The discrepancy between their results and ours can be attributed to the broader scale sampling by Zane et al. (1998), which included krill collected over a course of 3 years from different Southern Ocean regions.

Results obtained from Fu's Fs test $(F s=-23.62778$, $P<0.00850)$ indicated a population expansion of Antarctic krill and/or a selective sweep on mitochondrial DNA (Fu, 1997). These results corroborate previous inferences of a population expansion for Antarctic krill on the basis of data from the mitochondrial gene ND1 (Zane et al., 1998). The Bayesian Skyride analysis provided further insight into the population history of Antarctic krill, showing that the most recent doubling in abundance occurred within the last 100000 years (Figure 2). Assuming that the demographic history inferred herein is genuine and not solely due to mitochondrial DNA selection, these results represent the clearest evidence to date that Antarctic krill abundance might be correlated with climate change, as the cooler climate during the late Pleistocene may have promoted a krill population expansion, for example, through a proportionally larger area of sea ice acting as cover for larval krill

Our results indicate that the Antarctic krill stock within the Scotia Sea appears to be well mixed and diverse; this is encouraging considering the reported decrease in Antarctic krill abundance for the period 1976-2003 (Atkinson et al., 2008). However, the diversity reported in this study should not be considered as a cause for complacency, given predicted increases in global temperature and proposed, but not yet realized, increases in Antarctic krill fishing. Further population genetics research of krill at the swarm level should be encouraged to increase our understanding of krill population biology and to inform future policies for krill management.

\section{Conflict of interest}

The authors declare no conflict of interest. 


\section{Acknowledgements}

We thank the crew and scientists responsible for sampling Antarctic krill swarms on cruise JR82, Katrin Schmidt and Paul Giessler for their help in obtaining frozen krill samples, Chester Sands for comments on the sampling design, Laurent Excoffier and Erik Bloomquist for their advice with data analysis and Jon Seger and three anonymous reviewers for their comments on this paper. This study was supported by the BAS Q4 BIOREACH/BIOFLAME and Discovery 2010 core science programmes.

\section{References}

Ababneh F, Jermiin LS, Ma C, Robinson J (2006). Matched-pairs tests of homogeneity with applications to homologous nucleotide sequences. Bioinformatics 22: 1225-1231.

Ashton GV, Stevens MI, Hart MC, Green DH, Burrows MT, Cook EJ et al. (2008). Mitochondrial DNA reveals multiple northern hemisphere introductions of Caprella mutica (Crustacea, Amphipoda). Mol Ecol 17: 1293-1303.

Atkinson A, Siegel V, Pakhomov EA, Rothery P, Loeb V, Ros RM et al. (2008). Oceanic circumpolar habitats of Antarctic krill. Mar Ecol Prog Ser 362: 1-23.

Atkinson A, Siegel V, Pakhomov EA, Jessopp MJ, Loeb V (2009). A re-appraisal of the total biomass and annual production of Antarctic krill. Deep Sea Res Part I 56: 727-740.

Bargmann HE (1945). The development and life history of adolescent and adult krill, Euphausia superba. Discov Rep 23: 103-176.

Buchholz F, Watkins JL, Priddle J, Morris DJ, Ricketts C (1996). Moult in relation to some aspects of reproduction and growth in swarms of Antarctic krill, Euphausia superba. Mar Biol 127: 201-208.

Bucklin A, Wiebe PH, Smolenack SB, Copley NJ, Beaudet JG, Bonner KG et al. (2007). DNA barcodes for species identification of euphausiids (Euphausiacea, Crustacea). J Plankton Res 29: 483-493.

Buhay JE (2009). 'COI-like' sequences are becoming problematic in molecular systematic and DNA barcoding studies. J Crustac Biol 29: 96-110.

Clark AG, Whittam TS (1992). Sequencing errors and molecular evolutionary analysis. Mol Biol Evol 9: 744-752.

Crandall ED, Frey MA, Grosberg RK, Barber PH (2008). Contrasting demographic history and phylogeographical patterns in two Indo-Pacific gastropods. Mol Ecol 17: 611-626.

Drummond AJ, Rambaut A (2007). BEAST: Bayesian evolutionary analysis by sampling trees. BMC Evol Biol 7: 214.

Excoffier L, Smouse PE, Quattro JM (1992). Analysis of molecular variance inferred from metric distances among DNA haplotypes: application to human mitochondrial DNA restriction data. Genetics 131: 479-491.

Excoffier L, Laval G, Schneider S (2005). Arlequin (version 3.0): an integrated software package for population genetics data analysis. Evol Bioinform Online 1: 47-50.

Fevolden SE, Schneppenheim R (1989). Genetic homogeneity of krill (Euphausia superba Dana) in the Southern Ocean. Polar Biol 9: 533-539.

Fu YX (1997). Statistical tests of neutrality of mutations against population growth, hitchhiking and background selection. Genetics 147: 915-925.

Godlewska M (1996). Vertical migrations of krill (Euphausia superba Dana). Pol Arch Hydrobiol 43: 9-63.
Guindon S, Gascuel O (2003). A simple, fast, and accurate algorithm to estimate large phylogenies by maximum likelihood. Syst Biol 52: 696-704.

Ho JW, Adams CE, Lew JB, Matthews TJ, Ng CC, ShahabiSirjani A et al. (2006). SeqVis: visualization of compositional heterogeneity in large alignments of nucleotides. Bioinformatics 22: 2162-2163.

Kaliszewska ZA, Seger J, Rowntree VJ, Barco SG, Benegas R, Best PB et al. (2005). Population histories of right whales (Cetacea: Eubalaena) inferred from mitochondrial sequence diversities and divergences of their whale lice (Amphipoda: Cyamus). Mol Ecol 14: 3439-3456.

Lessios HA (2008). The great American schism: divergence of marine organisms after the rise of the Central American Isthmus. Annu Rev Ecol Evol Syst 39: 63-91.

Lin R-C, Yeung CK-L, Li S-H (2008). Drastic post-LGM expansion and lack of historical genetic structure of a subtropical fig-pollinating wasp (Ceratosolen sp. 1) of Ficus septica in Taiwan. Mol Ecol 17: 5008-5022.

Marr JWS (1962). The natural history and geography of the Antarctic krill. Discov Rep 32: 33-464.

Minin VN, Bloomquist EW, Suchard MA (2008). Smooth Skyride through a rough Skyline: Bayesian coalescent-based inference of population dynamics. Mol Biol Evol 25: 1459-1471.

Muths D, Jollivet D, Gentil F, Davoult D (2009). Large-scale genetic patchiness among NE Atlantic populations of the brittle star Ophiothrix fragilis. Aquat Biol 5: 117-132.

Nei M, Li WH (1979). Mathematical model for studying genetic variation in terms of restriction endonucleases. Proc Natl Acad Sci USA 76: 5269-5273.

Nei M (1987). Molecular Evolutionary Genetics. Columbia University Press: New York.

Patarnello T, Bargelloni L, Varotto V, Battaglia B (1996). Krill evolution and the Antarctic Ocean currents: evidence of vicariant speciation as inferred by molecular data. Mar Biol 126: 603-608.

Posada D (2008). jModelTest: phylogenetic model averaging. Mol Biol Evol 25: 1253-1256.

Rambaut A, Drummond AJ (2007) Tracer v1.4 Available at http:/ / beast.bio.ed.ac.uk/Tracer.

Ramos-Onsins SE, Rozas J (2002). Statistical properties of new neutrality tests against population growth. Mol Biol Evol 19: 2092-2100

Tarling GA, Shreeve RS, Hirst AG, Atkinson A, Pond DW, Murphy EJ et al. (2006). Natural growth rates in Antarctic krill (Euphausia superba): I improving methodology and predicting intermolt period. Limnol Oceanogr 51: 959-972.

Thorpe SE, Heywood KJ, Stevens DP, Brandon MA (2004). Tracking passive drifters in a high-resolution ocean model: implications for interannual variability of larval krill transport to South Georgia. Deep Sea Res Part I 51: 909-920.

Watkins JL (1986). Variations in the size of Antarctic krill, Euphausia superba Dana, in small swarms. Mar Ecol Prog Ser 31: 67-73.

Zane L, Ostellari L, Maccatrozzo L, Bargelloni L, Battaglia B, Patarnello T (1998). Molecular evidence for genetic subdivision of Antarctic krill (Euphausia superba Dana) populations. Proc R Soc B 265: 2387-2391.

Zwickl DJ (2006). Genetic algorithm approaches for the phylogenetic analysis of large biological sequence datasets under the maximum likelihood criterion. Ph D. dissertation. The University of Texas at Austin.

Supplementary Information accompanies the paper on Heredity website (http://www.nature.com/hdy) 